\title{
Murine amniotic fluid stem cells contribute mesenchymal but not epithelial components to reconstituted mammary ducts
}

\author{
Petra AB Klemmt, Vida Vafaizadeh, Bernd Groner ${ }^{*}$
}

\begin{abstract}
Introduction: Amniotic fluid harbors cells indicative of all three germ layers, and pluripotent fetal amniotic fluid stem cells (AFSs) are considered potentially valuable for applications in cellular therapy and tissue engineering. We investigated whether it is possible to direct the cell fate of AFSs in vivo by transplantation experiments into a particular microenvironment, the mammary fat pad. This microenvironment provides the prerequisites to study stem cell function and the communication between mesenchymal and epithelial cells. On clearance of the endogenous epithelium, the ductal tree can be reconstituted by the transfer of exogenously provided mammary stem cells. Analogously, exogenously provided stem cells from other tissues can be investigated for their potential to contribute to mammary gland regeneration.
\end{abstract}

Methods: We derived pluripotent murine AFSs, measured the expression of stem cell markers, and confirmed their in vitro differentiation potential. AFSs were transplanted into cleared and non cleared fat pads of immunocompromised mice to evaluate their ability to assume particular cell fates under the instructive conditions of the fat-pad microenvironment and the hormonal stimulation during pregnancy.

Results: Transplantation of AFSs into cleared fat pads alone or in the presence of exogenous mammary epithelial cells caused their differentiation into stroma and adipocytes and replaced endogenous mesenchymal components surrounding the ducts in co-transplantation experiments. Similarly, transplantation of AFSs into fat pads that had not been previously cleared led to AFS-derived stromal cells surrounding the elongating endogenous ducts. AFSs expressed the marker protein $\alpha$-SMA, but did not integrate into the myoepithelial cell layer of the ducts in virgin mice. With pregnancy, a small number of AFS-derived cells were present in acinar structures.

Conclusions: Our data demonstrate that the microenvironmental cues of the mammary fat pad cause AFSs to participate in mammary gland regeneration by providing mesenchymal components to emerging glandular structures, but do not incorporate or differentiate into ductal epithelial cells.

\section{Introduction}

The developing fetus is surrounded by a protective layer of liquid, the amniotic fluid. This fluid provides mechanical protection as well as nutrients required for fetal growth and well-being and contains cells derived from embryonic and extraembryonic tissues [1]. The initial cultures of amniotic fluid cells comprise a heterogeneous mixture of cell types [2,3], and differentiated cells, progenitor cells, and embryonic-like stem cells have

\footnotetext{
* Correspondence: groner@em.uni-frankfurt.de
Georg-Speyer-Haus, Institute for Biomedical Research, Paul-Ehrlich-Str. 42-44,

* Correspondence: groner@em.uni-frankfurt.de 60596 Frankfurt, Germany
}

() 2010 Groner et al.; licensee BioMed Central Ltd. This is an open access article distributed under the terms of the Creative Commons

been detected [4]. These cells exhibit differences in their adherence to culture plates. During prolonged culture, these cells acquire a more-homogeneous appearance and resemble fibroblast-like cells. The different origins of these cells can be determined with immunohistochemistry. The majority of amniotic fluid cells appear fibroblastoid, are rapidly proliferating, and co-express keratins and vimentin [5-7]. Cells derived from backflush cultures of amniocentesis specimens, obtained for prenatal genetic-screening procedures, were used for in vitro differentiation assays. They initially demonstrated multipotency, and the expression of embryonic 
stem cell markers suggested the presence of a pluripotent subpopulation $[4,8,9]$.

In the meantime, it has been shown that amniotic fluidderived stem cells (AFSs) are able to differentiate into neurogenic lineages and thus contribute to the ectodermal layer; into osteoblasts, fibroblasts, adipocytes, chondrocytes, and endothelial cells, as part of the mesodermal lineage, and into hepatocytic cells as part of the endodermal lineage. These cell-differentiation programs were triggered by culturing the AFSs in a wide spectrum of different media [10]; for example, basic fibroblast growth factor (bFGF), epidermal growth factor (EGF), and $N$-ethylmaleimide-sensitive factor (NSF-1) were required to induce the neuronal differentiation [11], and indomethacin, dexamethasone, methyl-3-isobutylxanthine, and insulin were added to the medium to achieve adipogenesis [8]. The multipotent nature of AFSs and the possibilities of differentiating them into diverse cell types have made them interesting candidates for therapeutic applications.

We investigated the plasticity of murine AFSs and their potential to adapt to a particular microenvironment. For this purpose, we performed transplantation experiments of AFSs into the mammary fat pads of mice.

The mammary gland is a highly dynamic organ; its development is mainly postnatal, and its tissue composition changes in a characteristic fashion during puberty, pregnancy, lactation, and involution [12]. The branching ducts are of ectodermal origin and consist of a single layer of luminal epithelial cells surrounded by a contractile layer of myoepithelial cells, causing milk ejection with oxytocin induction. The myoepithelial cells are in direct contact with an extracellular basement membrane and contribute its components. They are instrumental for the establishment of ductal polarity and lumen formation [13]. The mammary ducts are embedded in an adipose stroma of mesodermal origin, which consists mainly of adipocytes, but also comprises fibroblasts, endothelial cells, and immune cells involved in the regulation of mammogenesis [14]. The ducts infiltrate the adipose stroma during puberty, and the alveolar structures that grow out during pregnancy are interspersed with islands of adipocytes. During lactation, the adipocytes lose most of their fat content, but persist as long projections in the interstitial space. A layer of fibrous connective tissue is present around the ducts and the secretory alveoli [15].

The cyclical nature of cell proliferation, differentiation, and apoptosis and the regenerative capacity of the epithelium during successive cycles of pregnancy, lactation, and involution require the presence and activity of stem cells $[16,17]$. A unique feature of mammary stem cells is their ability to reconstitute the ductal component of the mammary gland with transplantation into cleared fat pads [18]. This is a distinct property of the mammary system and is comparable to the reconstitution of the hematopoietic system by stem cell transplantation [19]. In these circumstances, stem cells are functionally selected by their reconstitution ability in vivo.

Transplantation of stem cells from adult male seminiferous tubules and of neural stem cells from embryonic or adult brain into fat pads was shown to adopt functional mammary epithelial cell traits, but only in conjunction with normal, stem cell-depleted mammary epithelial cells after pregnancy. These results indicate that tissue-specific signals from the mammary stroma cooperate with signals originating from differentiated mammary epithelial cells and that these combined influences are capable of directing the fate of stem cells from heterologous organs [20,21].

The ready availability of AFSs and the expectations that these cells might become a convenient source for therapeutic applications caused us to investigate their adaptability to a particular organ microenvironment and their potential to contribute to mammary gland structures. Cultured AFSs by themselves or mixed with primary mammary epithelial cells were introduced into cleared and noncleared fat pads of mice to gauge their plasticity toward mammary epithelial differentiation. Our results indicate that the signals operating in this microenvironment trigger an exclusive mesenchymal differentiation program of transplanted AFSs, irrespective of the presence of mammary epithelial cells in virgin mice. The AFSs proliferate and persist during mammary gland development, interact closely with endogenous or transplanted mammary epithelial cells, and provide stromal components and adipocytes surrounding the ductal structures. Under the hormonal influence of pregnancy, a very small number of AFS-derived cells appear present in acinar structures between the basement membrane and the myoepithelium.

\section{Materials and methods \\ Mice}

C57BL/6-Tg (ACTB-EGFP) and B6;129S-GT(ROSA) 26Sor/J (Rosa26) from Jackson Laboratories (Charles River, Sulzfeld, Germany) were used as donor mice for amniotic fluid or mammary epithelial cell isolation. Female RAG2 KO mice (BALB/cA-RAG2KO, IL-2R $\gamma \mathrm{KO}$ ) from Central Institute for Experimental Animals (CIEA, Kawasaki, Japan) were used as hosts for the transplantation studies. All mice were maintained in a pathogen-free facility, and the experimental procedures were approved by the Animal Welfare office of the Regierungspräsidium Darmstadt.

Isolation and culture of AFSs from murine amniotic fluid Amniotic fluid was collected from pregnant donor mice at embryonic day 13.5 by rupturing the yolk sac with a $27-$ gauge needle, and recovered cells were cultured in $35-\mathrm{mm}$ 
dishes in AmnioMax complete (Invitrogen, Darmstadt, Germany) by using a two-stage culture method [9]. In brief, after an initial expansion of 5 days, the nonadherent amniotic fluid cells in the supernatant were collected, centrifuged for $5 \mathrm{~min}$ at $300 \mathrm{~g}$, and replated in AmnioMax complete for a further 5 days, by which they acquired an adherent phenotype. Established AFS lines were passaged at a dilution of 1:4 to $1: 8$ in DMEM supplemented with $10 \%$ fetal calf serum (FCS), 50 units $/ \mathrm{ml}$ and $50 \mu / \mathrm{ml}$ penicillin-streptomycin, and $2 \mathrm{~m} M$ L-glutamine (DMEM complete; all from Invitrogen, Darmstadt, Germany).

\section{FACS analysis}

For flow-cytometric analysis, $5 \times 10^{5}$ cells were fixed for $5 \mathrm{~min}$ in 3\% para-formaldehyde (PFA, Sigma-Aldrich, Munich, Germany), washed with PBS (PAA Laboratories $\mathrm{GmbH}$, Cölbe, Germany), and incubated with $1 \mu$ fluorescence-conjugated antibodies in $200 \mu \mathrm{l}$ PBS against CD24, CD29, CD44, CD117, Sca-1, CD49f, CD90.2 (all BD Biosciences, Heidelberg, Germany), and CD31 and CD45 (Biolegend, BIOZOL Diagnostica Vertrieb $\mathrm{GmbH}$, Eching, Germany) or their respective IgG controls for 20 min in the dark. After two washing steps with PBS, cells were analyzed by using a FACS Scan (Becton Dickinson, Heidelberg, Germany) flow cytometer with CellQuest software (Becton Dickinson, Heidelberg, Germany).

\section{Immunocytochemistry}

AFS cultures were fixed in 3\% PFA, permeabilized (10 $\mathrm{mM}$ HEPES, $200 \mathrm{~m} M$ sucrose, $3 \mathrm{mM} \mathrm{MgCl} 2,50 \mathrm{mM} \mathrm{NaCl}$, $0.5 \%$ Triton $\mathrm{X}-100,0.2 \% \mathrm{NaN}_{3}$, all from Sigma-Aldrich, Munich, Germany) and blocked in 3\% BSA (SigmaAldrich, Munich, Germany). Specific antigens were detected by incubation with antibodies to $\alpha$-keratin 18 (clone KS18.04; Progen Biotechnik GmbH, Heidelberg, Germany, ready-to-use), $\alpha$-keratin 14 (clone AF64; Covance, HiSS Diagnostics GmbH, Germany, 1:200), $\alpha$-vimentin (ab45939; abCam, Cambridge, UK, 1:500), $\alpha$-CD44-CyChrome (clone IM7; BD Biosciences, Heidelberg, Germany, 1:60), and $\alpha-S M A$ (clone 1A4; SigmaAldrich, Munich, Germany, 1:500) for $1 \mathrm{~h}$ at RT in $0.1 \%$ BSA followed by incubation with $10 \mu / \mathrm{ml}$ of donkey anti-mouse AlexaFluor546-conjugated IgG (Invitrogen, Darmstadt, Germany) or donkey anti-rabbit AlexaFluor488-conjugated IgG (Invitrogen, Darmstadt, Germany) for 45 min at RT in the dark and mounted in Mowiol containing $1.5 \mu \mathrm{g} / \mathrm{ml}$ DAPI. Staining was assessed by using a Nikon Eclipse TE300 microscope and NIS Elements AR imaging software (Nikon, Düsseldorf, Germany).

\section{Differentiation protocols}

AFS were cultured to confluence in DMEM complete before the addition of adipogenic supplements $(1 \mu M$ dexamethasone, $0.5 \mathrm{~m} M$ isobutylmethylxanthine, $5 \mu / \mathrm{ml}$ Insulin, and $60 \mu M$ indomethacin, all from SigmaAldrich, Munich, Germany), osteogenic supplements (100 $\mathrm{n} M$ dexamethasone, $10 \mathrm{~m} M \beta$-glycerolphosphate, and $50 \mu M 2$-phospho ascorbic acid, all from SigmaAldrich, Munich, Germany) or epithelial supplements (10 ng/ml keratinocyte growth factor, $10 \mathrm{ng} / \mathrm{ml}$ hepatocyte growth factor, $60 \mathrm{ng} / \mathrm{ml}$ insulin-like growth factor II (all from BIOZOL Diagnostica Vertrieb GmbH, Eching, Germany), and20 $\mathrm{ng} / \mathrm{ml}$ epidermal growth factor (BD Biosciences, Heidelberg, Germany), ) in DMEM containing $10 \%$ FCS and antibiotics for 14 days. The differentiation medium was changed every 4 days. The differentiation potential for adipogenesis was assessed with the visualization of lipid vacuoles by using Oil Red O staining (Sigma-Aldrich, Munich, Germany); the deposition of mineralized calcium accumulation during osteogenesis was detected by Alizarin Red S staining (Sigma-Aldrich, Munich, Germany), and the epithelial character was confirmed with Ayoub Shklar staining [22].

\section{Measurement of mRNA expression with RT-PCR}

Total RNA was extracted from cultured, noninduced or differentiated AFSs by using NucleoSpin RNA II (Macherey \& Nagel, Düren, Germany) according to the manufacturer's instructions. RNA was then reverse transcribed by using Superscript II reverse transcriptase (Invitrogen, Darmstadt, Germany), and RT-PCR was performed by using standard protocols. The amplified DNA fragments were subjected to $1.5 \%$ agarose gel electrophoresis.

\section{Isolation and culture of mammary epithelial cells}

Mammary glands were isolated from 10-week-old virgin female C57BL/6-Tg (ACTB-EGFP) or B6;129S-GT(ROSA) $26 S o r / J$, mice as previously described [17]. The organoids were purified with pulse centrifugation and plated on a thin gel of collagen type I (BD Biosciences, Heidelberg, Germany) in Ham's F-12 (PAA Laboratories GmbH, Cölbe, Germany) supplemented with $5 \%$ FCS, $5 \mu / \mathrm{ml}$ insulin (Sigma-Aldrich, Munich, Germany), $1 \mu / \mathrm{ml}$ hydrocortisone (Sigma-Aldrich, Munich, Germany), $5 \mathrm{ng} / \mathrm{ml}$ EGF, 50 units/ml-50 $\mu / \mathrm{ml}$ penicillin-streptomycin, and $50 \mu / \mathrm{ml}$ gentamicin (Invitrogen, Darmstadt, Germany). Primary cultures were passaged once, 3 to 4 days after plating before transplantation.

\section{Three-dimensional cell-culture system}

The 3D culture of AFSs and MECs was performed as described [23]. In brief, cells were trypsinized and resuspended in assay medium (DMEM/F12 containing 2\% FCS, $0.5 \mu / \mathrm{ml}$ hydrocortisone, $100 \mathrm{ng} / \mathrm{ml}$ choleratoxin (Sigma-Aldrich, Munich, Germany), $10 \mu / \mathrm{ml}$ insulin) at a concentration of 25,000 cells/ml. Eight-chamber glass 
slides (Nunc, VWR International GmbH, Darmstadt, Germany) were coated with $45 \mu$ l growth factor reduced Matrigel (BD Biosciences, Heidelberg, Germany) per well and left to gel at $37^{\circ} \mathrm{C}$ for $15 \mathrm{~min}$. The cells were mixed 1:1 with assay medium supplemented with $4 \%$ Matrigel and $10 \mathrm{ng} / \mathrm{ml} \mathrm{EGF}$, and $400 \mu \mathrm{l}$ of this mixture was added to each well. Assay medium supplemented with $2 \%$ Matrigel and $5 \mathrm{ng} / \mathrm{ml}$ EGF was replaced every 4 days. Acini formation was assessed after 5 days by using a Nikon Eclipse TE300 microscope and NIS Elements AR imaging software (Nikon, Düsseldorf, Germany) and after 10 days by using a Leica TCS SL confocal microscope and Leica Confocal software version 2.61 (Leica Microsystems, Wetzlar, Germany). Immunostaining of $3 \mathrm{D}$ cultures was performed as described [24] with antibodies to $\alpha$-vimentin (ab45939, abCam, Cambridge, UK; 1:500) and $\alpha-S M A$ (clone 1A4, Sigma-Aldrich, Munich, Germany; 1:500).

\section{Cell transplantation into mammary fat pads}

The 3-week-old Rag $2^{-/-}$recipient mice were anesthetized, and the endogenous epithelium was removed from both number 4 inguinal mammary glands. AFSs alone or mixed with MECs were resuspended in $10 \mu \mathrm{l}$ cold Matrigel and injected into the remaining fat pad, cleared of its epithelial component. The hosts were analyzed 8 weeks later. The $\mathrm{GFP}^{+}$outgrowths were visualized by using a Leica MZ16F stereomicroscope and LAS version 2.8 (Leica Microsystems, Wetzlar, Germany), fixed in $4 \%$ formalin for $1 \mathrm{~h}$ at $4^{\circ} \mathrm{C}$, and incubated in X-gal staining solution $\left(10 \mathrm{mM} \mathrm{K}_{3} \mathrm{Fe}(\mathrm{CN})_{6}\right.$, $10 \mathrm{mM} \mathrm{K}{ }_{4} \mathrm{Fe}(\mathrm{CN})_{6}, 0.2 \% \mathrm{NP}-40,0.1 \%$ Triton $\mathrm{X}-100$, and $1 \mathrm{mg} / \mathrm{ml} \mathrm{X-Gal,} \mathrm{all} \mathrm{from} \mathrm{Sigma-Aldrich,} \mathrm{Munich,} \mathrm{Ger-}$ many) overnight at $37^{\circ} \mathrm{C}$ to visualize $\beta$-galactosidasepositive outgrowth. Stained glands were rinsed in PBS, photographed, postfixed in $4 \%$ formalin, and embedded in paraffin.

\section{Immunohistochemistry}

Immunohistochemical analysis was performed on formalin-fixed paraffin sections $(5 \mu \mathrm{m})$, cleared in xylene, and rehydrated through an alcohol series. Antigen retrieval by using $10 \mathrm{~m} M$ sodium citrate buffer, $\mathrm{pH} \mathrm{6}$, was performed for all antibodies except $\alpha$-SMA. IHC-HRP staining was performed by using the Envision detection system Peroxidase/DAB (Dako, Hamburg, Germany) and the M.O.M. kit (Vector Laboratories, BIOZOL Diagnostica Vertrieb $\mathrm{GmbH}$, Eching, Germany) according to the manufacturer's instructions. Slides were counterstained with hematoxylin or Nuclear fast red, dehydrated, and mounted with Assistent-Histokitt. Staining was assessed by using a Leica DMLS microscope (Leica Microsystems, Wetzlar, Germany) and LAS version 2.8. For IHC-IF staining, the sections were blocked in $0.1 \mathrm{mg} / \mathrm{ml}$ goat anti-mouse IgG (Jackson ImmunoResearch, Dianova, Hamburg, Germany) and 10\% FCS in PBS for $1 \mathrm{~h}$. Primary antibodies were incubated in 1\% FCS overnight at $4^{\circ} \mathrm{C}$ followed by incubation with $10 \mu / \mathrm{ml}$ goat anti-mouse AlexaFluor488-conjugated IgG (Invitrogen, Darmstadt, Germany), donkey anti-rabbit AlexaFluor647-conjugated IgG (Invitrogen, Darmstadt, Germany), and donkey antigoat AlexaFluor594-conjugated IgG (Invitrogen, Darmstadt, Germany) for $45 \mathrm{~min}$ at RT in the dark and mounted in Mowiol containing $1.5 \mu \mathrm{g} / \mathrm{ml}$ DAPI. Staining was assessed by using a Leica TCS SP 5 confocal microscope and LAS AF imaging software (Leica Microsystems, Wetzlar, Germany). Images were processed by using Imaris (Bitplane, Zurich, Switzerland). The sources of antibodies used in this study: $\alpha$-GFP (2555; Cell Signaling, New England Biolabs GmbH, Frankfurt, Germany, 1:800); $\alpha$-Keratin14 (clone AF64; Covance, HiSS Diagnostics GmbH, Germany, 1:500); $\alpha-S M A$ (clone 1A4; Sigma, Munich, Germany, 1:500), $\alpha$-vimentin (clone C20; Santa Cruz, Heidelberg, Germany, 1:500), $\alpha-c / E B P \alpha$ (clone 14AA; Santa Cruz, Heidelberg, Germany, 1:50), and $\alpha$-laminin $\beta 1$ (clone C19; Santa Cruz, Heidelberg, Germany, 1:50).

\section{Results}

Amniotic fluid-derived cells exhibit an epithelial phenotype and are multipotent

We collected amniotic fluid from the yolk sac of mice at day 13.5 of pregnancy and isolated amniotic fluid stem cells (AFSs) by using a two-stage culture model [9]. Nonadherent amniotic fluid cells after 5 days of culture were recovered from the medium and replated for a further 5 days (Figure 1a). During this period, they became adherent. In total, we established 20 AFS lines from different donors. The rapidly adhering cells isolated during the initial plating period of 5 days underwent growth senescence within three passages. Morphologically, undifferentiated AFSs obtained during the second culture period appear spindle shaped and resemble fibroblasts.

We characterized the freshly isolated and cultured AFSs with respect to the expression of genes that are indicative of stem-cell phenotypes and measured the mRNA levels of Oct-4, SSEA-1, Nanog, ZFX, Tbx3, and Bmi-1. These genes have been found to maintain cells in a pluripotent, undifferentiated state, and Nanog is a master regulator of pluripotency in embryonic stem cells. The expression of Nanog, $Z f x$, and $T b x 3$ persists at least for 15 passages, and $B m i-1$ was detected up to passage 3 (Figure 1b). Flow cytometry was used to visualize the expression of stem cell-associated surface markers. The cultured AFSs express predominantly markers of mesenchymal stem cells (CD44, Sca-1, CD29, CD49f, and CD90) but lack marker expression of the hematopoietic compartment (CD31 and CD45) 


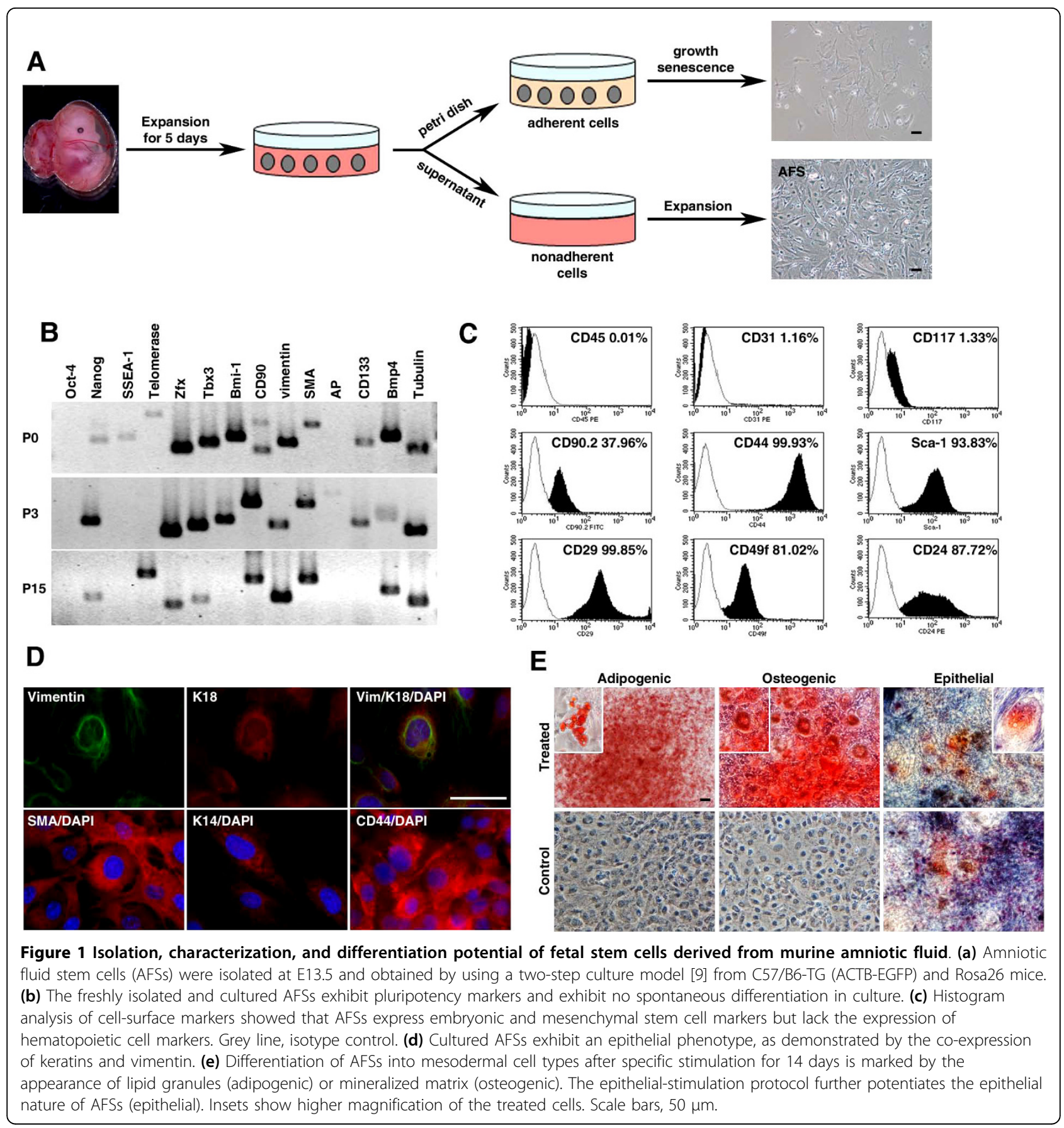

(Figure 1c). Furthermore, our cultured murine AFSs express $C D 117$ to a similar degree as that reported for human AFS lines [8]. Additionally, they express CD24, a pan-epithelial marker in the mouse mammary gland [25]. The derived AFSs are epithelial in nature, as shown by their expression of the luminal epithelial marker keratin 18 (K18) and the myoepithelial marker keratin 14 (K14). At the same time, they express mesenchymal cells markers, (for example, vimentin and $\alpha$-smooth muscle actin
$(\alpha-S M A)$ and the mesenchymal stem-cell marker $C D 44$ (Figure 1d)).

The hallmark of adult stem cells is their ability to produce progeny of different lineages. We confirmed the multipotency of the cultured murine AFS lines by their differentiation capacity into various cell types in vitro, after 14 days of exposure to specific induction conditions (Figure 1e). Differentiation of AFSs in adipogenic medium [9] resulted in the formation of lipid vacuoles 
verified by Oil red $\mathrm{O}$ staining. Osteogenic differentiation medium [9] caused the formation of functional osteoblasts characterized by the deposition of mineralized calcium, as shown by Alizarin Red $S$ staining. The differentiation of AFSs in epithelial medium [26] resulted in the enhancement of the epithelial keratin expression, as shown by Ayoub Shklar staining, a modified Mallory connective tissue stain for keratins [22].

\section{AFSs closely associate with mammary epithelial cells in the formation of acini in 3D culture}

The interaction of AFSs with primary mammary epithelial cells (MECs) was tested in co-culture experiments. AFSs derived from C57BL/6-Tg (ACTB-EGFP) donors were either mixed with single-cell suspensions of MECs isolated from Rosa26 mice before plating or added to adherent MECs organoid cultures. Interestingly, MECs formed islands after plating surrounded by $\mathrm{GFP}^{+} \mathrm{AFS}$ or $\mathrm{GFP}^{+}$AFS were found in close association with the organoids (data not shown). We extended these observations and performed three-dimensional culture (3D) experiments in the Matrigel microenvironment. MECs display distinct morphogenesis in 3D cultures; they resemble features of the mammary gland architecture in vivo [27]. When grown in the presence of basement membrane components, MECs form hollow spherical, acini-like structures with apicobasal polarization formed by a single layer of polarized, growth-arrested cells [23]. AFS lines derived from Rosa26 donors grown under the same conditions in the 3D Matrigel culture system begin to form spherical structures within 5 days of culture, which extend to form tubular networks after 10 days (Figure 2a). This is comparable to the cordlike structures obtained from human AFSs, pre-differentiated toward the endothelial lineage [28]. Immunostaining of the tubular structures showed the expression of the mesenchymal markers vimentin and $\alpha-S M A$ (Figure $2 \mathrm{~b}$ ). Closer investigation of the spherical structures revealed the formation of a lumen after 10 days (Figure 2b, inset).

We investigated whether AFSs can associate or cooperate with MECs and integrate into acinar structures formed by MECs. Equal numbers of AFSs, derived from Rosa26 donors, and MECs, derived from GFP transgenic donors, C57BL/6-Tg (ACTB-EGFP), were cultured under conditions that allow $3 \mathrm{D}$ differentiation. The formation of acini was monitored 10 days after plating (Figure 2c). We observed the emergence of branching tubular structures formed by AFSs surrounding small clusters of $\mathrm{GFP}^{+} \mathrm{MECs}$ (Figure 2c, upper panel) or spheres formed by $\mathrm{GFP}^{+}$MECs with a hollow central lumen surrounded by a layer of cells contributed by AFS (Figure 2c, middle panel). The AFSs branched off the acini and surrounded additional $\mathrm{GFP}^{+}$MECs (Figure 2c, lower panel). Immunostaining experiments of the obtained structures with $\alpha-S M A$ suggest that AFSs closely associate with MECs and contribute $\alpha-S M A^{+}$ mesenchymal cells to emerging acini (Figure $2 \mathrm{~d}$ ) but do not integrate into the MEC cell layer.

\section{The microenvironment of the mammary fat pad triggers AFS differentiation into mesenchymal cells}

Mammary epithelial outgrowths can be observed in cleared mammary fat pads transplanted with ductal fragments or dispersed mammary cells. This procedure can be used to identify mammary stem cells and to study their properties. We used the fat-pad microenvironment to investigate the regenerative potential of AFS lines derived from GFP-transgenic mice, C57BL/6-Tg (ACTBEGFP). We compared amniotic fluid cells (AFs) that became adherent within the initial culture period of 5 days and AFS that became adherent after only 5 days of culture. AF cells and AFSs $\left(2 \times 10^{5}\right.$ each $)$ were inoculated into epithelium-divested inguinal mammary fat pads of 3-week-old recipient Rag $2^{-/ \text {- }}$ female mice, and outgrowths were analyzed 8 weeks later with fluorescence microscopy of whole mounts. Only AFSs that became adherent after day 5 of the isolation procedure were able to implant and grow in cleared mammary fat pads (Figure 3a, top panel). The $\mathrm{GFP}^{+}$outgrowths derived from four independent transplantation experiments with different GFP $^{+}$AFS lines yielded mainly connective tissue and contained a mixture of stromal cells and adipocytes (Figure 3a, lower panel). Differentiation into mammary epithelium was not detected. The AFS differentiation capacity was not influenced by pregnancy hormones or tissue remodeling during involution. This observation was confirmed with immunohistochemistry on sections derived from the outgrowths with antibodies specific for GFP, K14, and $\alpha-S M A$ (Figure $3 \mathrm{~b}$ ). $K 14$ and $\alpha-S M A$ expression colocalized with the expression of GFP. The $\mathrm{GFP}^{+}$cells originated from the transplanted AFSs and provide connective tissue components composed of stromal cells and adipocytes. Immunohistochemical analysis showed that the cells originating from the amniotic fluid stem cells $\left(\mathrm{GFP}^{+}\right)$ expressed stromal cell markers $(\alpha-S M A$ and vimentin) and adipocyte marker $(C / E B P \alpha)$ (Figure $3 \mathrm{c})$.

We also examined the possibility that MECs might be required for the participation of AFSs in ductal reconstitution, analogous to the observations made with the transplantation of neural stem cells [21]. It is conceivable that the co-transplantation of mammary epithelial cells can alter the signals acting on AFSs. Equal numbers of MECs and AFS were combined $\left(10^{5}\right.$ each) and immediately inoculated into cleared fat pads of 3-weekold Rag $2^{-1 /}$ recipients. Both combinations (AFSs derived from Rosa26 donors combined with $\mathrm{GFP}^{+} \mathrm{MECs}$ and 

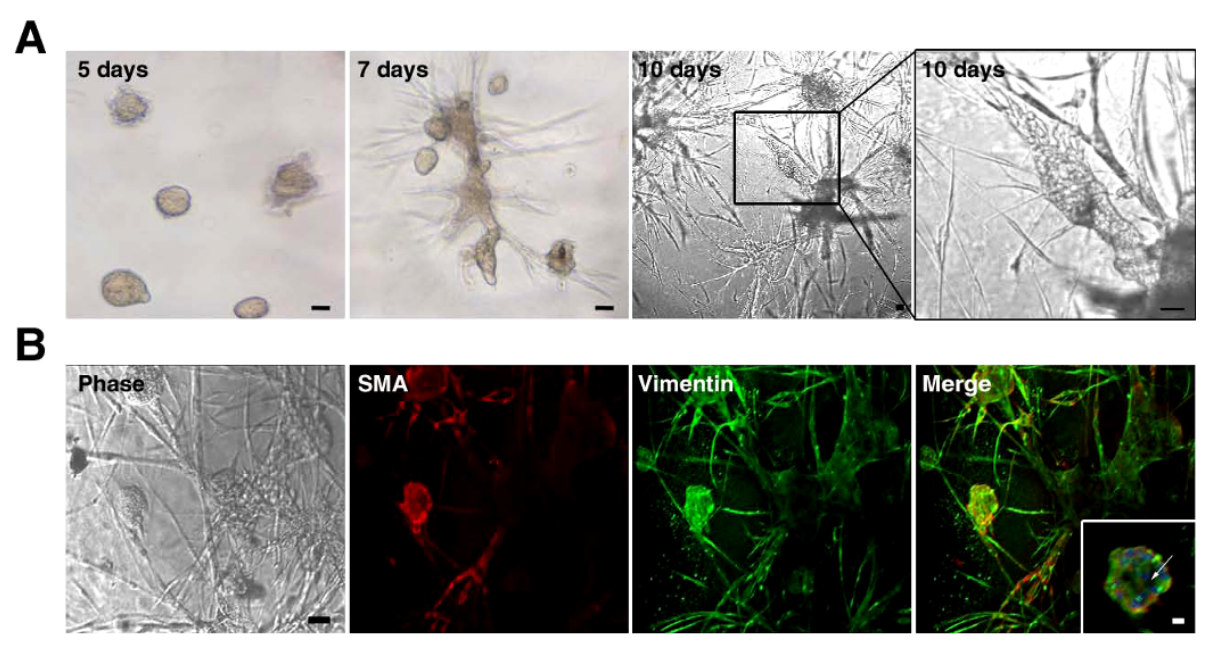

C
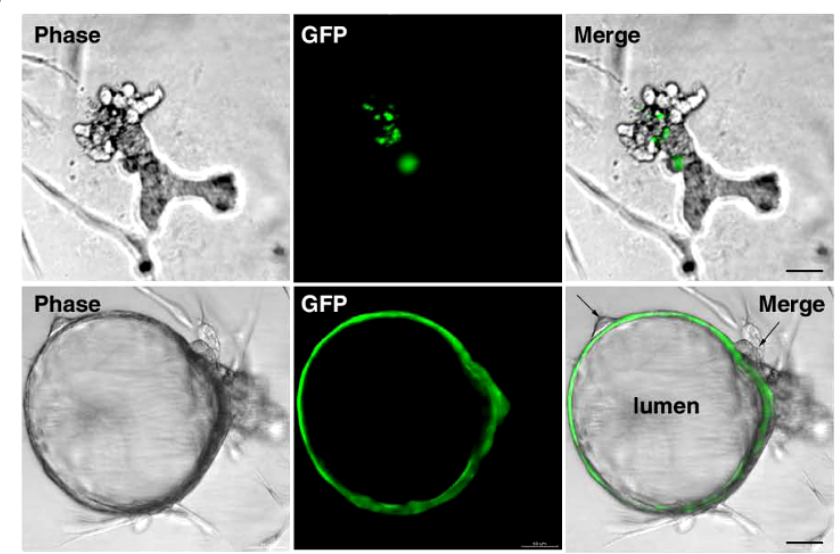

D
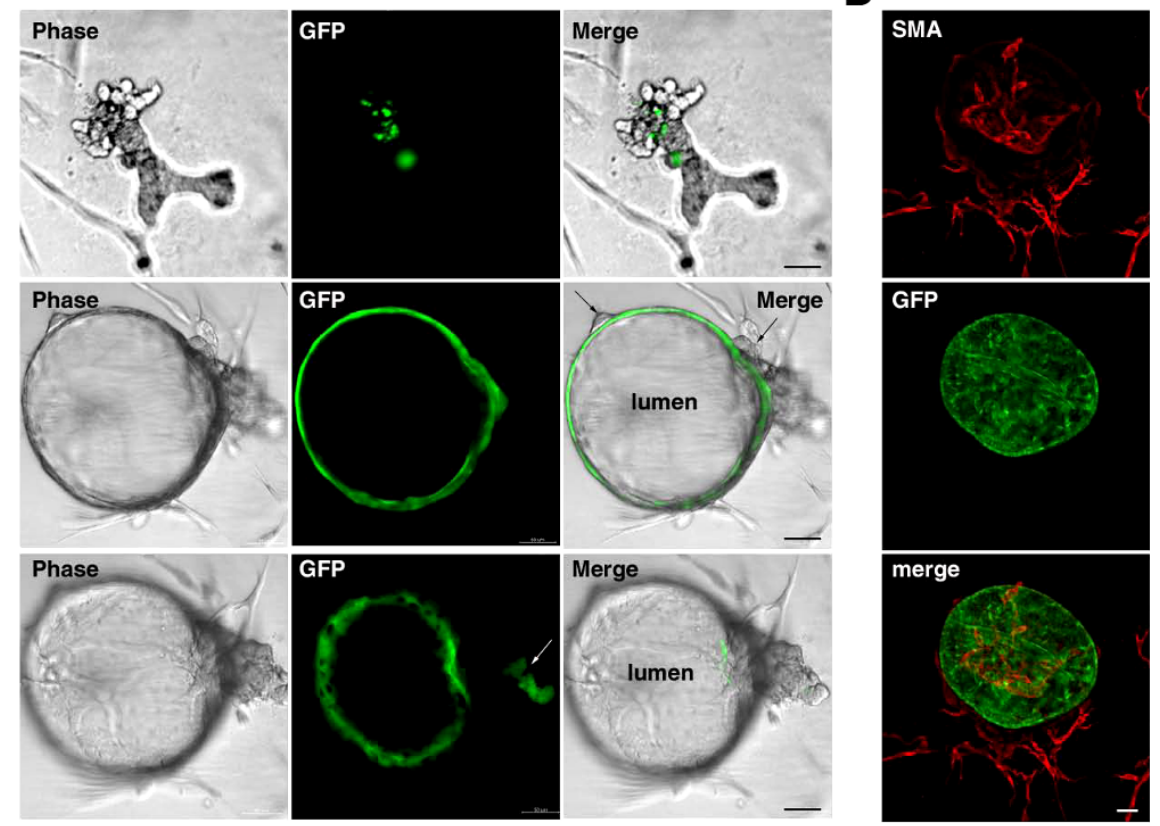

Figure 2 Amniotic fluid stem cells (AFSs) form spheres and tubular structures in 3D cultures. AFSs alone or equal numbers of AFSs and MECs derived from GFP transgenic C57/B6-TG (ACTB-EGFP) mice were subjected to 3D differentiation and monitored for up to 10 days after plating. (a) AFS-derived progeny form spherical structures 5 days after plating and extend to a branched tubular network in 3D Matrigel culture. (b) These structures are positive for $\alpha$-SMA and vimentin. Inset shows a section through a spherical structure. (c) We observed the emergence of tubular structures formed by AFSs surrounding GFP' MECs (upper panel) or spheroids formed from GFP ${ }^{+}$MECs with a hollow central lumen surrounded by a layer of cells contributed by AFSs (middle panel) and side branches surrounding GFP' MECs (lower panel). (d) AFSs closely associated with GFP ${ }^{+}$MECs express $\alpha$-SMA. Scale bars, $50 \mu \mathrm{m}$, and for inset, $30 \mu \mathrm{m}$.

AFS derived from C57BL/6-Tg (ACTB-EGFP) donors combined with $\mathrm{LacZ}^{+}$MECs) were analyzed in virgin, pregnant, and postlactation mice (Figure 4). Eight weeks after transplantation, mice were either mated or analyzed as virgin mice. Whole mounts of the resulting glands were subjected to fluorescence microscopy before staining with X-Gal. The AFS progeny contributed ringlike structures around the growing ducts (Figure 4a), reminiscent of the results obtained in the $3 \mathrm{D}$-culture experiments in virgin mice. Histologic analysis revealed that AFS-derived cells contributed stromal components surrounding the mammary epithelial ducts in virgin mice, but did not incorporate into the epithelial cell layers (Figure $4 \mathrm{~b}$ ). The differentiation stimulus during pregnancy did not alter the cell fate of the majority of transplanted AFSs (Figure 4c). They contributed $\mathrm{GFP}^{+}$ stromal components tightly associated with emerging alveoli (Figure 4c, upper panel). Histologic analysis by 


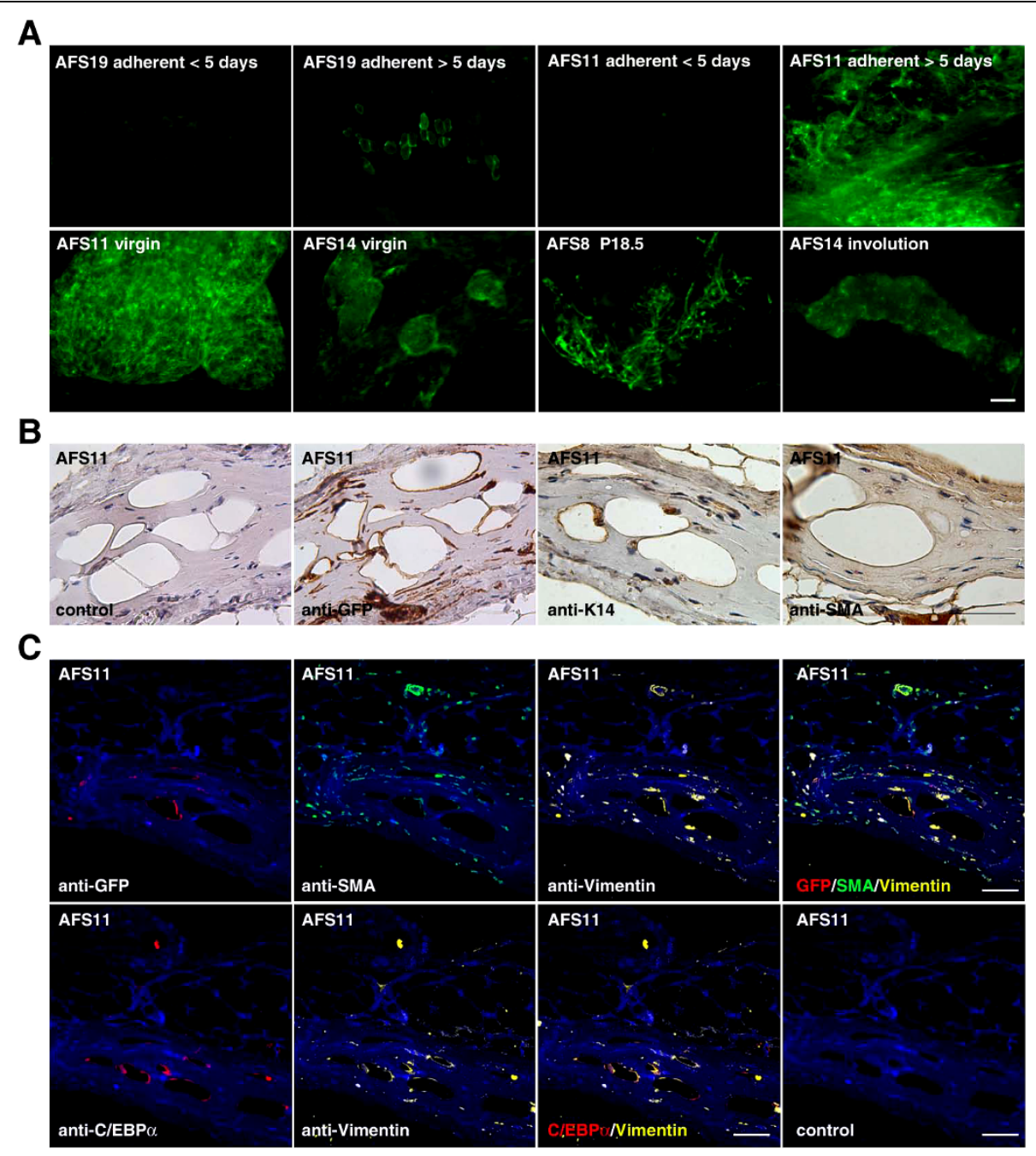

Figure 3 Cell-fate tracing of transplanted amniotic fluid stem cells (AFSs) into cleared fat pads. (a) The $2 \times 10^{5}$ AF-derived cells (rapidly adherent within 5 days) and AFSs (slowly adherent, after 5 days of culture) derived from various donors were transplanted into 3-week-old recipient mice and analyzed 8 weeks later as whole mounts. Only the slowly adhering cells selected in the second culture step are able to engraft and can be classified as AFSs. (b) Histologic analysis confirms that the GFP ${ }^{+}$AFSs survived and differentiated into adipocytes and connective tissue. Scale bar for whole mounts and tissue sections is $50 \mu \mathrm{m}$, (Blue) X-Gal stain or hematoxylin, (Pink) Nuclear fast red, and (Brown) DAB staining.

using IHC-IF and antibodies specific for GFP, $\alpha-S M A$, and laminin $\beta 1$ revealed that a small number of $\mathrm{GFP}^{+}$ AFSs can be detected associated with alveolar structures (Figure 4c, lower panel). Thus AFS progeny do not incorporate into ductal structures in virgin mice, but can be found in acinar structures during pregnancy. The engrafted AFSs persisted during involution (data not shown).

\section{AFSs inoculated into the mammary fat pads exhibit a} remarkable migratory potential

Previous studies indicated that transplanted AFSs can migrate away from the injection site [29], and, if injected intraperitoneally or intravenously, are able to diffuse systemically and to integrate into various tissues [30,31]. We investigated whether AFSs inoculated into the cleared mammary fat pads are able to migrate to other organs. AFSs obtained from Rosa26 mice express the $L a c Z$ gene, and their progeny can be monitored by whole-mount staining of different organs. On injection of these cells into the mammary fat pad, Lac $\mathrm{Z}^{+}$progeny of AFSs were detected in several organs. The cells inoculated into the number 4 fat pad migrated to the number 3 fat pad on the same side and also were found in healing suture wounds (data not shown). The gastrointestinal tract contained the largest number of $\mathrm{LacZ}^{+}$ 


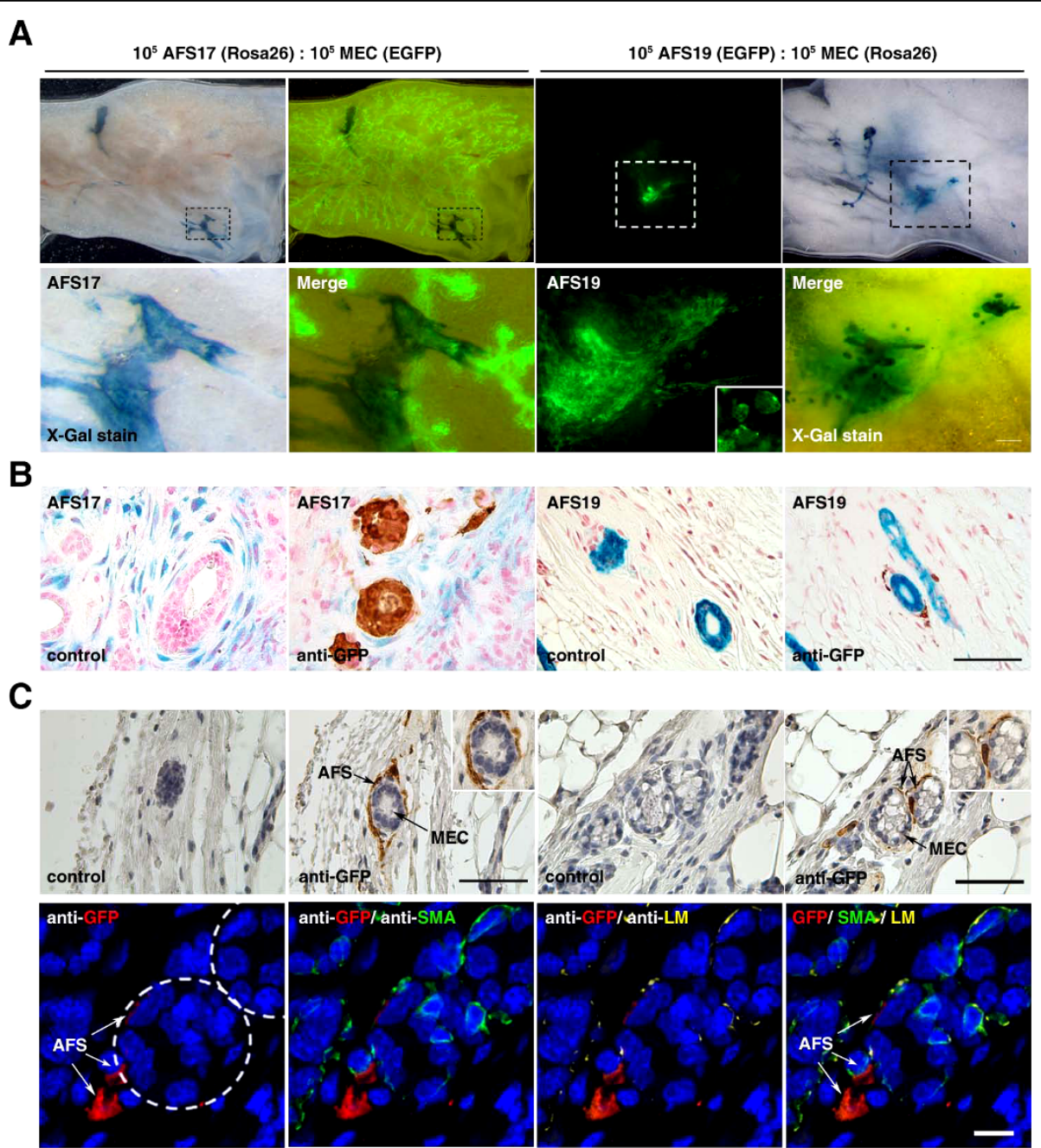

Figure 4 Cell-fate tracing of amniotic fluid stem cells (AFSs) in co-transplantation with MECs into cleared fat pads. (a) AFSs were transplanted in a 1:1 ratio with supporting MECs ( $10^{5}$ each) and analyzed 8 weeks later. X-Gal-stained whole mounts from virgin mice show that AFS-derived progeny surround the developing ductal structures. (b) Histologic analysis of whole mounts inoculated with AFSs and MECs confirms that AFSs form a stromal cell layer around the ductal outgrowth but do not integrate into the myoepithelial or luminal cell layer in virgin mice. (c) Histologic analysis of whole mounts at pregnancy day 18.5 inoculated with GFP ${ }^{+}$AFSs and MECs shows that the stromal cell layer derived from GFP ${ }^{+}$AFSs exhibit no adverse effects on alveologenesis. Insets show higher magnification. Scale bar for whole mounts and tissue sections is $50 \mu \mathrm{m}$. A few $\alpha$-SMA $A^{+}$and GFP ${ }^{+}$AFS-derived cells can be detected within the alveolar unit, as shown by laminin $\beta 1$ staining. The circles indicate alveolar units. Scale bar is $10 \mathrm{~mm}$. (Blue) X-Gal stain or hematoxylin, (Pink) Nuclear fast red, and (Brown) DAB staining.

cells, followed by the liver and the lung. The AFSderived cells integrated into the normal tissue architecture (Figure 5).

\section{The AFSs contribute stromal components to emerging ducts}

To investigate whether the AFSs can contribute mesodermal components to endogenous epithelium, we injected $10^{5}$ AFSs derived from a Rosa26 donor into noncleared fat pads of 3-week-old Rag $2^{-/-}$recipients in close proximity to the nipple (Figure 6). Glands were excised 2 weeks later, subjected to X-Gal staining, and counterstained with carmine alum to visualize the ductal epithelium. Whole-mount analysis demonstrates engraftment and massive proliferation of the transplanted AFSs (Figure 6a). The LacZ ${ }^{+}$cells fill the fat pad around the injection site and surround the endogenous, elongating ducts, reminiscent of the co-transplantation experiments with exogenously added MECs. Immunohistochemistry of paraffin sections confirmed the presence of $\mathrm{LacZ}^{+}$ cells in the stromal compartment of the extending endogenous ducts and around terminal end buds (TEBs), but also revealed areas of adipocyte differentiation. The LacZ $^{+}$cells expressed the myoepithelial marker $\alpha-S M A$, but did not incorporate into the myoepithelial cell layer (Figure 6b). Based on these observations, we conclude 


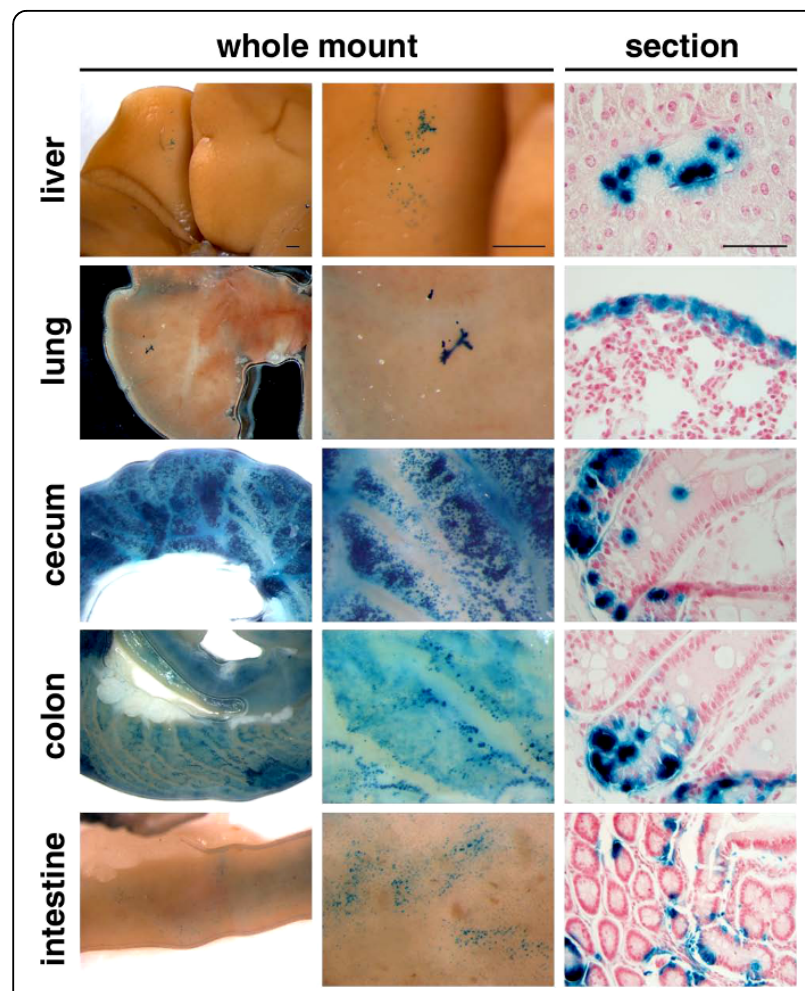

Figure 5 Amniotic fluid stem cells (AFS)-derived cells migrate to other organs. The $10^{5}$ AFSs derived from a Rosa26 donor were injected into cleared fat pads of 3-week-old recipient mice and analyzed 14 weeks later. X-Gal-stained whole mounts demonstrate the presence of $\mathrm{LacZ}^{+}$cells in several organs. Tissue sections show the localization of AFS-derived LacZ ${ }^{+}$cells between hepatocytes, adjacent to airway epithelial cells, and in the mucosa of the gastrointestinal tract. Scale bar for whole mounts is $500 \mu \mathrm{m}$, and for sections, $50 \mu \mathrm{m}$, (Blue) X-Gal stain and (Pink) Nuclear fast red staining.

that the stimulus and signals provided by the fat pad only trigger differentiation programs of AFS into mesenchymal cells types, despite their original, partially epithelial nature.

\section{Discussion}

Amniotic fluid-derived stem cells (AFSs) are a most versatile cell population and are interesting for conceptual and practical purposes. Conceptually, they can be used to investigate the culture conditions and the molecular signals required for stem cells to assume particular cell fates. Practically, AFS, triggered by defined cues to differentiate into certain cell types, can potentially be used in transplantation studies for tissue repair or tissue reconstitution. Despite their obvious potential value, a routine procedure has yet to be established for the derivation of AFSs, their growth in culture, and the maintenance of their pluripotent nature.

Several protocols differ considerably from each other. A single-step cultivation method has been used in

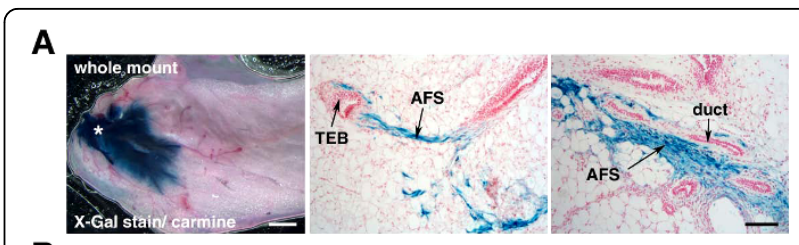

B

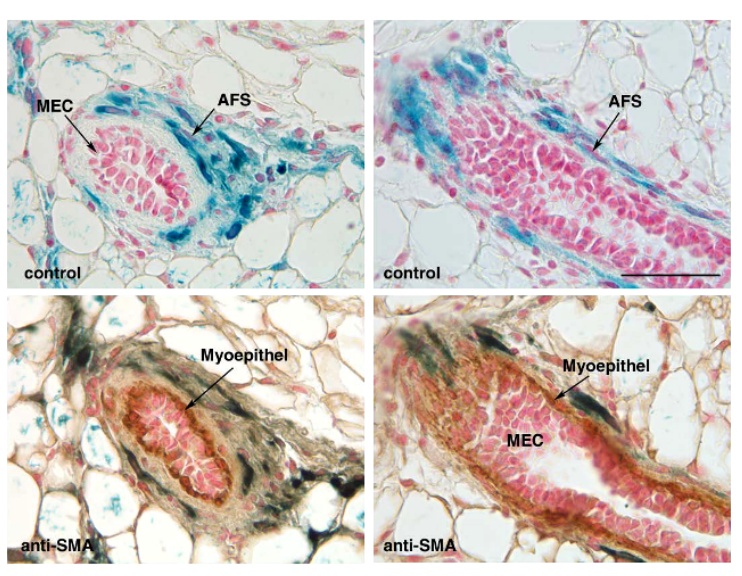

Figure 6 Amniotic fluid stem cells (AFS)-derived cells contribute stromal components for extending ducts. The $10^{5}$ AFSs derived from a Rosa26 donor were injected into noncleared fat pads of 3-week-old recipient mice and analyzed 2 weeks later. (a) Whole-mount analysis and tissue sections of X-Gal-stained whole mounts demonstrate the presence of $\mathrm{LacZ}^{+}$cells in the stroma of extending ducts. (b) AFS-derived LacZ ${ }^{+}$cells retain the expression of $\alpha$-SMA but did not integrate into the myoepithelial cell layer. Scale bar for whole mount is 1,000 mm, and for tissue sections, $50 \mu \mathrm{m}$; (*) injection site, (Blue) X-Gal stain, (Pink) Carmine alum or Nuclear fast red staining and (Brown) DAB staining.

which AFSs are obtained after 7 days of cultivation without medium changes [29,32-34]. A two-stage culture method favors the isolation of Oct-4-positive AFSs [9] and the isolation of CD117-positive AFSs [8,30]. These methods use different growth media and isolation methods and yield AFSs of distinct phenotypes. In our experiments, we made use of the two-stage culture method developed for the isolation and enrichment of human AFSs obtained by amniocentesis for prenatal diagnosis [9] and applied this method to the investigation of murine AFSs. During this procedure, AFSs slowly adapt to adherent growth and can be classified according to their morphologic appearance. The cells maintain their ability to differentiate into adipogenic, osteogenic, myogenic, endothelial, neurogenic, and hepatic cells in vitro $[8,9]$. We consistently observed that the amniotic fluid-derived stem cells became adherent between days 5 and 7 after plating. These cells showed unlimited proliferation and the maintenance of their differentiation potential. Conversely, the amniotic fluid cells that became adherent within the first 5 days after plating underwent rapid growth senescence and did not 
engraft in cleared mammary fat pads, irrespective of the presence of supporting mammary epithelial cells.

The majority of the murine AFSs used in this study appeared fibroblastoid, proliferated rapidly, and coexpressed keratins and vimentin, confirming their partial epithelial nature. This is in contrast to bone marrowderived mesenchymal stem cells (BM-MSCs), which need special medium supplements to differentiate toward the epithelial lineage [26] and proliferate at a lower rate than AFSs [35]. The multipotency of the murine AFSs was confirmed by in vitro differentiation assays. Adipogenic, osteogenic, and epithelial lineages were observed.

In this study, we investigated whether the signals provided by the mammary microenvironment are sufficient to redirect the cell fate of multipotent AFSs into mammary epithelium. We also probed the influence of exogenously added mammary epithelial cells on the differentiation potential of AFS in both in vitro and in vivo assays. We investigated the in vitro morphogenesis of AFSs in a 3D-on-top culture system, a test assay to monitor mammary acinar architecture [23]. In this assay system, we observed the formation of spherelike structures with a small lumen by AFSs followed by the emergence of branched tubular structures that mimic the sprouting of predifferentiated AFSs toward endothelium [28] or endothelial precursor cells [36]. Interestingly, the 3D coculture of AFSs and MECs yielded similar results. MECs were embedded in tubes formed by AFS or developed acinar structures surrounded by a layer of AFSs extending away from the spherical structures. It appears that the AFSs provide stromal components to the glandular epithelium and form a scaffold around MECs.

We also analyzed the differentiation potential of AFSs in vivo by transplantation experiments into cleared and noncleared mammary fat pads. Previous studies using cells from seminiferous tubules [20] or neural stem cells [21] of male WAP-Cre/Rosa26 reporter mice showed that the mammary microenvironment could alter the intrinsic cell fate of exogenously provided stem cells toward the mammary epithelial cell lineage after pregnancy. Mesenchymal stem cells from different sources have the potential to differentiate into a variety of cells under the influence of certain microenvironments. Despite the partial epithelial nature of the cultured AFSs, we consistently observed that the progeny of AFSs adopt mesenchymal cell properties after transplantation into the mammary microenvironment. This is in contrast to the engraftment capacity of cells from seminiferous tubules or neural stem cells, which did not persist in the absence of supporting mammary epithelial cells $[20,21]$. The mammary fat pad is of mesodermal origin and provides factors that cause AFS differentiation toward mesenchymal cell types. The morphogenetic capacity of the ectoderm-derived mammary epithelium strongly depends on the microenvironmental clues provided by the fat pad. It is, therefore, interesting to note that AFSs contribute stromal components to emerging ducts that have no adverse effect on mammary epithelial repopulation capacity. The engrafted AFSs continued to express myoepithelial markers but did not integrate into the luminal or myoepithelial layer of the emerging ducts in virgin mice.

Myoepithelial cells play an important role in the morphogenetic events during gland development and influence the proliferation, survival, and differentiation of luminal cells and modulate stromal-epithelial interactions [37]. Interestingly, we observed the presence of a small number of AFS-derived cells in close proximity to emerging alveoli during pregnancy.

At this point, we cannot distinguish between two possible interpretations. It is conceivable that AFSs are intrinsically limited in their differentiation potential, that they are unable to assume the mammary epithelial cell fate, and that the stroma-induced transdifferentiation potential of fetal or adult tissue might be restricted to cell fates of the same germ layer. For example, endoderm-derived pulmonary and pancreatic epithelia failed to exhibit morphogenetic responses to the adult mammary fat pad [38]. It is possible that our AFS lines emerged from a germ-layer lineage that is not conducive to differentiate into epithelium in response to the signals provided by the mammary fat pad, even in the presence of MECs. In concordance with this notion is the observation that neural stem cells [21] are able to differentiate into mammary epithelium, but only after cotransplantation with MECs depleted of endogenous stem cells and after the hormonal stimulus exerted during pregnancy. These cells are derived from the same germ layer as the mammary gland, the ectoderm that might account for this differentiation potential.

Alternatively, it is possible that the AFSs are not exposed to the proper signals required for mammary epithelial differentiation. The timing of the exposure and the cellular environment might contribute influences that we cannot control at present. This might explain the presence of a small number of AFS-derived cells within an alveolar unit during pregnancy. Interestingly, transplantation under the renal capsule indicates that mammary mesenchyme can be replaced by salivary [38] or prostatic [39] mesenchyme to facilitate mammary epithelial morphogenesis. Based on these observations, it is conceivable that the AFS-derived mesenchyme supports mammary epithelial morphogenesis. In contrast to cleared mammary fat pads, the renal capsule is a highly vasculogenic graft site that supports the engraftment of human mammary tissue and other heterologous tissue components. For this reason, it has been used in many tissuetransplantation studies [40]. 
The results obtained in our in vivo studies are consistent with the observations we made in the in vitro experiments. AFSs participate in the ductal outgrowth, persist over long periods in association with the luminal and myoepithelial cells, but do not become part of the ductal epithelial structures. They contribute mesenchymal components to the glandular epithelium and are remarkably motile. Because of this motile phenotype, it is not too surprising that AFSderived cells are occasionally incorporated into mammary epithelial structures during pregnancy. The inoculated AFSs proliferate, and their progeny can be detected in a number of distant organs several weeks after transplantation. Lac $\mathrm{Z}^{+}$cells were found in other epithelial tissue, such as the lung and the mucosa of the gastrointestinal tract. $\mathrm{LacZ}^{+}$cells at these sites were incorporated into the tissues. Previous reports have shown that BM-MSCs and AFS can contribute to airway-epithelium restoration $[30,31,41]$, and BM-MSCs migrate and participate in gastrointestinal tract mucosa regeneration after damage [42]. The ability of AFSs to migrate and engraft at distant sites and to incorporate into the gastrointestinal mucosa suggests that the signals of the microenvironment can influence the cell fate of AFSs.

\section{Conclusions}

The observation that undifferentiated AFSs survive in a permissive environment and have the potential to differentiate into multiple cell types makes them interesting candidates for use as therapeutic cells and for their application in tissue repair. Here we demonstrated that the signals provided by the mammary microenvironment and the communication between AFSs and mammary epithelium favor the formation of supportive stroma for the extending ductal epithelium, but do not cause the epithelial differentiation of AFSs. AFSs exhibit a remarkably motile phenotype, and their ability to migrate from the mammary fat pad to other organs might shed light on mechanisms of metastasis formation.

\section{Abbreviations \\ $\alpha$-SMA: Alpha smooth muscle actin; AFSs: amniotic fluid stem cells; BM- MSCs: bone marrow-derived mesenchymal stem cells; MECs: mammary epithelial cells; TEB: terminal end bud.}

\section{Acknowledgements}

This work was supported by grants from the Deutsche Forschungsgemeinschaft (DFG, GR 536/9-2, and GR 536/11-1). We thank the CIEA (Kawasaki, Japan) for the Rag $2^{-/-} \gamma^{-/-}$mice.

\section{Authors' contributions}

PK developed ideas, designed and conducted the experiments, and wrote and edited the manuscript. W conducted and contributed to the analysis of data and edited the manuscript. BG developed ideas and edited the manuscript.

\section{Competing interests}

The authors declare that they have no competing interests.
Received: 1 April 2010 Revised: 17 June 2010 Accepted: 7 July 2010 Published: 7 July 2010

\section{References}

1. Underwood MA, Gilbert WM, Sherman MP: Amniotic fluid: not just fetal urine anymore. J Perinatol 2005, 25:341-348.

2. Gosden CM: Amniotic fluid cell types and culture. Br Med Bull 1983, 39:348-354.

3. Sachs L, Serr DM, Danon M: Prenatal diagnosis of sex using cells from the amniotic fluid. Science 1956, 123:548.

4. Prusa AR, Marton E, Rosner M, Bernaschek G, Hengstschlager M: Oct-4expressing cells in human amniotic fluid: a new source for stem cell research? Hum Reprod 2003, 18:1489-1493.

5. Chen WW: Studies on the origin of human amniotic fluid cells by immunofluorescent staining of keratin filaments. J Med Genet 1982, 19:433-436.

6. Virtanen I, von Koskull H, Lehto VP, Vartio T, Aula P: Cultured human amniotic fluid cells characterized with antibodies against intermediate filaments in indirect immunofluorescence microscopy. J Clin Invest 1981, 68:1348-1355

7. Ochs BA, Franke WW, Moll R, Grund C, Cremer M, Cremer T: Epithelial character and morphologic diversity of cell cultures from human amniotic fluids examined by immunofluorescence microscopy and gel electrophoresis of cytoskeletal proteins. Differentiation 1983, 24:153-173.

8. De Coppi P, Bartsch G, Siddiqui MM, Xu T, Santos CC, Perin L, Mostoslavsky G, Serre AC, Snyder EY, Yoo JJ, Furth ME, Soker S, Atala A: Isolation of amniotic stem cell lines with potential for therapy. Nat Biotechnol 2007, 25:100-106.

9. Tsai M-S, Lee J-L, Chang Y-J, Hwang S-M: Isolation of human multipotent mesenchymal stem cells from second-trimester amniotic fluid using a novel two-stage culture protocol. Hum Reprod 2004, 19:1450-1456.

10. Parolini O, Soncini M, Evangelista M, Schmidt Dr: Amniotic membrane and amniotic fluid-derived cells: potential tools for regenerative medicine? Regen Med 2009, 4:275-291.

11. Mareschi K, Rustichelli D, Comunanza V, De Fazio R, Cravero C, Morterra G, Martinoglio B, Medico E, Carbone E, Benedetto C, Fagioli F: Multipotent mesenchymal stem cells from amniotic fluid originate neural precursors with functional voltage-gated sodium channels. Cytotherapy 2009, 11:534-547.

12. Hennighausen $L$, Robinson $\mathrm{GW}$ : Information networks in the mammary gland. Nat Rev Mol Cell Biol 2005, 6:715-725.

13. Sternlicht MD: Key stages in mammary gland development: the cues that regulate ductal branching morphogenesis. Breast Cancer Res 2006, 8:201.

14. Neville MC, Medina D, Monks J, Hovey RC: The mammary fat pad. J Mammary Gland Biol Neoplasia 1998, 3:109-116.

15. Wiseman BS, Werb Z: Stromal effects on mammary gland development and breast cancer. Science 2002, 296:1046-1049.

16. Stingl J, Eirew P, Ricketson I, Shackleton M, Vaillant F, Choi D, Li HI, Eaves $\mathrm{CJ}$ : Purification and unique properties of mammary epithelial stem cells. Nature 2006, 439:993-997.

17. Shackleton M, Vaillant F, Simpson KJ, Stingl J, Smyth GK, Asselin-Labat ML, Wu L, Lindeman GJ, Visvader JE: Generation of a functional mammary gland from a single stem cell. Nature 2006, 439:84-88.

18. Kordon EC, Smith GH: An entire functional mammary gland may comprise the progeny from a single cell. Development 1998 125:1921-1930.

19. Ott MG, Schmidt M, Schwarzwaelder K, Stein S, Siler U, Koehl U, Glimm H, Kuhlcke K, Schilz A, Kunkel H, Naundorf S, Brinkmann A, Deichmann A, Fischer M, Ball C, Pilz I, Dunbar C, Du Y, Jenkins NA, Copeland NG, Lüthi U, Hassan M, Thrasher AJ, Hoelzer D, von Kalle C, Seger R, Grez M: Correction of $\mathrm{X}$-linked chronic granulomatous disease by gene therapy, augmented by insertional activation of MDS1-EVI1, PRDM16 or SETBP1. Nat Med 2006, 12:401-409.

20. Boulanger CA, Mack DL, Booth BW, Smith GH: Interaction with the mammary microenvironment redirects spermatogenic cell fate in vivo. Proc Natl Acad Sci USA 2007, 104:3871-3876.

21. Booth BW, Mack DL, Androutsellis-Theotokis A, McKay RD, Boulanger CA Smith GH: The mammary microenvironment alters the differentiation repertoire of neural stem cells. Proc Natl Acad Sci USA 2008, 105:14891-14896. 
22. Ayoub P, Shklar G: A modification of the Mallory connective tissue stain as a stain for keratin. Oral Surg Oral Med Oral Pathol 1963, 16:580-581.

23. Debnath J, Muthuswamy SK, Brugge JS: Morphogenesis and oncogenesis of MCF-10A mammary epithelial acini grown in three-dimensional basement membrane cultures. Methods 2003, 30:256-268.

24. Lee GY, Kenny PA, Lee EH, Bissell MJ: Three-dimensional culture models of normal and malignant breast epithelial cells. Nat Methods 2007 , 4:359-365

25. Sleeman KE, Kendrick H, Ashworth A, Isacke CM, Smalley MJ: CD24 staining of mouse mammary gland cells defines luminal epithelial, myoepithelial/ basal and non-epithelial cells. Breast Cancer Res 2006, 8:R7

26. Paunescu V, Deak E, Herman D, Siska IR, Tanasie G, Bunu C, Anghel S, Tatu CA, Oprea TI, Henschler R, Rüster B, Bistrian R, Seifried E: In vitro differentiation of human mesenchymal stem cells to epithelial lineage. $J$ Cell Mol Med 2007, 11:502-508.

27. Mailleux A, Overholtzer M, Brugge J: Lumen formation during mammary epithelial morphogenesis: insights from in vitro and in vivo models. Cell Cycle 2008, 7:57-62.

28. Zhang P, Baxter J, Vinod K, Tulenko TN, Di Muzio PJ: Endothelial differentiation of amniotic fluid-derived stem cells: synergism of biochemical and shear force stimuli. Stem Cells Dev 2009, 18:1299-1308.

29. Cipriani S, Bonini D, Marchina E, Balgkouranidou I, Caimi L, Grassi Zucconi G, Barlati S: Mesenchymal cells from human amniotic fluid survive and migrate after transplantation into adult rat brain. Cell Biol Int 2007, 31:845-850.

30. Ghionzoli M, Cananzi M, Zani A, Rossi CA, Fascetti Leon F, Pierro A, Eaton S, De Coppi P: Amniotic fluid stem cell migration after intraperitoneal injection in pup rats: implication for therapy. Pediatr Surg Int 2010, 26:79-84.

31. Minoo P, Atala A, De Filippo RE, Warburton D: Human amniotic fluid stem cells can integrate and differentiate into epithelial lung lineages. Stem Cells 2008, 26:2902-2911.

32. Antonucci I, Pantalone A, De Amicis D, D'Onofrio S, Stuppia L, Palka G, Salini V: Human amniotic fluid stem cells culture onto titanium screws: a new perspective for bone engineering. J Biol Regul Homeost Agents 2009, 23:277-279.

33. Yoo YA, Kang MH, Kim BS, Kim JS, Seo JH: Sustained co-cultivation with human placenta-derived MSCs enhances ALK5/Smad3 signaling in human breast epithelial cells, leading to EMT and differentiation. Differentiation 2009, 77:450-461.

34. Kim J, Lee Y, Kim H, Hwang K, Kwon H, Kim S, Cho D, Kang S, You J: Human amniotic fluid-derived stem cells have characteristics of multipotent stem cells. Cell Proliferation 2007, 40:75-90.

35. Kunisaki SM, Fuchs JR, Steigman SA, Fauza DO: A comparative analysis of cartilage engineered from different perinatal mesenchymal progenitor cells. Tissue Eng 2007, 13:2633-2644.

36. Bagley RG, Walter-Yohrling J, Cao X, Weber W, Simons B, Cook BP, Chartrand SD, Wang C, Madden SL, Teicher BA: Endothelial precursor cells as a model of tumor endothelium: characterization and comparison with mature endothelial cells. Cancer Res 2003, 63:5866-5873.

37. Faraldo MM, Teuliere J, Deugnier MA, Taddei-De La Hosseraye I, Thiery JP, Glukhova MA: Myoepithelial cells in the control of mammary development and tumorigenesis: data from genetically modified mice. $J$ Mammary Gland Biol Neoplasia 2005, 10:211-219.

38. Sakakura T, Nishizuka Y, Dawe CJ: Capacity of mammary fat pads of adult $\mathrm{C} 3 \mathrm{H} / \mathrm{HeMs}$ mice to interact morphogenetically with fetal mammary epithelium. J Nat/ Cancer Inst 1979, 63:733-736.

39. Taylor RA, Wang H, Wilkinson SE, Richards MG, Britt KL, Vaillant $F$, Lindeman GJ, Visvader JE, Cunha GR, St John J, Risbridger GP: Lineage enforcement by inductive mesenchyme on adult epithelial stem cells across developmental germ layers. Stem Cells 2009, 27:3032-3042.

40. Parmar H, Cunha GR: Epithelial-stromal interactions in the mouse and human mammary gland in vivo. Endocr Relat Cancer 2004, 11:437-458.

41. Ortiz LA, Gambelli F, McBride C, Gaupp D, Baddoo M, Kaminski N, Phinney DG: Mesenchymal stem cell engraftment in lung is enhanced in response to bleomycin exposure and ameliorates its fibrotic effects. Proc Natl Acad Sci USA 2003, 100:8407-8411.

42. Wei Y, Nie Y, Lai J, Wan YJ, Li Y: Comparison of the population capacity of hematopoietic and mesenchymal stem cells in experimental colitis rat model. Transplantation 2009, 88:42-48.

\section{doi:10.1186/scrt20}

Cite this article as: Klemmt et al:: Murine amniotic fluid stem cells contribute mesenchymal but not epithelial components to reconstituted mammary ducts. Stem Cell Res Ther 2010 1:20.

\section{Submit your next manuscript to BioMed Central and take full advantage of:}

- Convenient online submission

- Thorough peer review

- No space constraints or color figure charges

- Immediate publication on acceptance

- Inclusion in PubMed, CAS, Scopus and Google Scholar

- Research which is freely available for redistribution

Submit your manuscript at www.biomedcentral.com/submit
Biomed Central 